\section{Protracted cerebral circulatory arrest and cortical electrical silence coexisting with preserved respiratory drive and flexor motor response}

Editor-Recent case reports of ventilated patients who had been confirmed brain dead based on neurological criteria and subsequently resumed spontaneous ventilation while awaiting organ retrieval prompted some authors to advocate a broader use of ancillary tests as a safeguard in the diagnosis of brain stem death (BSD). ${ }^{1}$ The topic remains highly controversial. $^{2}$ It is our opinion that the reported specificity of tests confirming cerebral circulatory arrest may be overestimated. Only patients who already fulfil BSD criteria are usually tested. If the test 'confirms' brain death, support is withdrawn (or organs are taken for donation), thus realizing a self-fulfilling prophecy. It should come as no surprise that 'confirmatory' tests come with a reported specificity of $100 \% .^{34}$

We were recently involved in the care of a comatose 45-yr-old male with a large right-sided intracerebral haemorrhage (ICH). Monitoring initiated on admission included

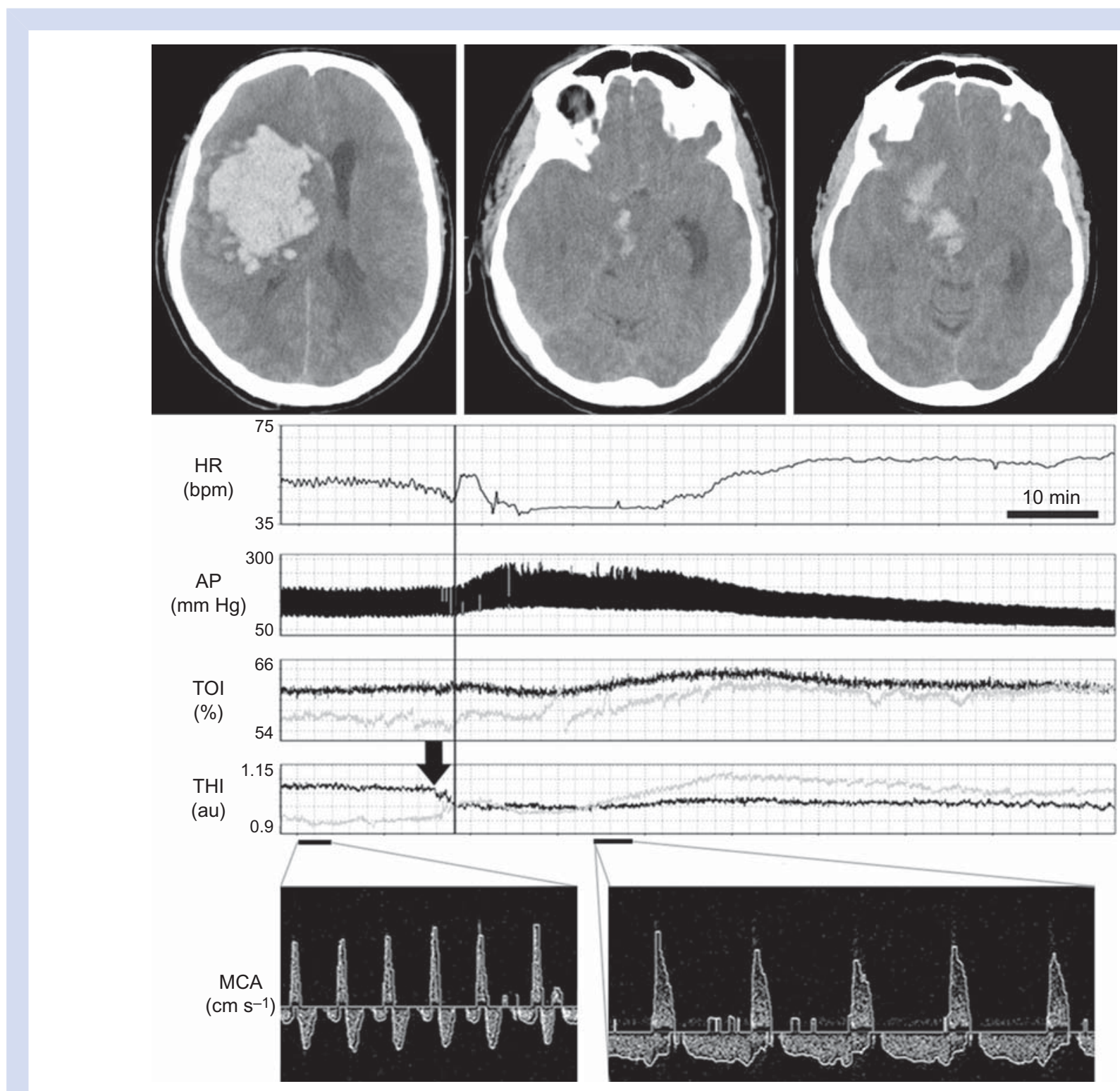

Fig 1 CT head: a large right-sided fronto-parietal ICH with extension into the basal ganglia, right cerebral peduncle, and midbrain is seen. Subfalcine herniation and effacement of basal cisterns is visible. Multimodal neuromonitoring: the vertical line demarcates the onset of the Cushing reflex with bradycardia and hypertension (top two panels). Before the AP increase, asymmetrical changes in THI, thought to represent significant intracranial volume shifts, are visible (arrow). There is a global increase in TOI thought to represent venous outflow obstruction or reduced metabolic demand on both sides (bilaterally). TCD recording form the middle cerebral artery demonstrates diastolic reversal of flow before (left panel) and during (right panel) the Cushing reflex. AP, arterial pressure; HR, heart rate; MCA, middle cerebral artery; TOI, tissue oxygenation index; THI, tissue haemoglobin index. 
transcranial Doppler (TCD), near-infrared spectroscopy (NIRS) with calculation of tissue oxygen index (TOI) and bispectral index (BIS) EEG. Four hours after admission, pupils became dilated and fixed and repeat computed tomography (CT) showed progression of the ICH deemed unsurvivable by the medical team (Fig. 1). Sedation was stopped and palliation and withdrawal of treatment was discussed with family.

Twenty-six hours after ictus, TCD showed bilateral diastolic reversal of flow in all basal cerebral arteries, including the basilar artery. Simultaneously, the unprocessed EEG obtained on the BIS monitor was isoelectric. In patients with abolished brain stem reflexes, these TCD and BIS findings would independently be considered to be 'confirmatory' of death with a reported specificity of $100 \% .^{3}{ }^{4}$ NIRS also demonstrated a marked reduction in cortical oxygenation. Despite confirmation of cerebral circulatory arrest and cortical electrical silence, the patient was still making active respiratory efforts with flexor motor posturing.

Six hours later, multimodal monitoring documented a Cushing reflex characterized by hypertension (290/120 mm $\mathrm{Hg}$ ) and bradycardia (Fig. 1). During that time, TCD signals remained unchanged and EEG remained isoelectric, while NIRS seemed to indicate a bilateral increase in frontal cerebral oxygenation with a reduction in right-to-left asymmetry. The patient continued to breathe spontaneously with flexor motor response. Organ support was withdrawn and the patient died shortly after.

This unusual case highlights three important points. The first is of a physiological nature. Cushing reflex has previously been interpreted as a homeostatic baroreflex which can restore cerebral blood flow (CBF) during episodes of intracranial hypertension. ${ }^{5}$ In this case, extreme hypertension did not result in any improvement in cerebral circulation, arguing against this interpretation and supporting the view that the Cushing reflex can be a paroxysmal pre-mortal phenomenon. The second significant observation is that NIRS monitoring can be potentially misleading during extreme haemodynamic events. Importantly, NIRS estimates blood flow at a depth of $\sim 0.5-1 \mathrm{~cm}$ in the frontal cortex, making extrapolation to global CBF potentially erroneous. Thirdly, in the context of the absence of cerebral electrical activity and persistent cerebral circulatory arrest, the observed increase in TOI during the Cushing reflex could be interpreted as the accumulation of oxygenated blood due to obstructed venous outflow or reduced metabolic activity due to widespread cerebral ischaemia. In the absence of EEG and TCD monitors, the increase in TOI observed during extreme hypertension could have been misinterpreted as a partial restoration of CBF.

In the UK, the diagnosis of brain death in adult patients remains based on comprehensive clinical examination, the exclusion of potential confounders (including a sufficient time of observation), and confirmation of complete loss of brain stem function. ${ }^{6}$ The final and most important observation in this case is that ultrasonographic and EEG evidence of cerebral circulatory arrest and cortical electrical silence can coexist with active motor responses and preserved respiratory drive for many hours.

\section{Declaration of interest}

None declared.

\section{Funding}

This project was supported by the National Institute of Health Research Brain Repair Centre (Cambridge University Hospitals Foundation Trust) Neuroscience Theme. K.P.B. received a scholarship from the Fearnside Trust, UK, and Marry and Clifford Corbridge Trust of Robinson College, UK. M.J.H.A. received a travel grant from the European Federation of Neurological Societies and is supported by the Netherlands Organisation for Health Research and Development.

\author{
K. P. Budohoski* \\ M. J. H. Aries \\ P. J. Kirkpatrick \\ A. Lavinio \\ Cambridge, UK \\ *E-mail: kpb26@cam.ac.uk
}

1 Roberts DJ, MacCulloch KA, Versnick EJ, Hall RI. Should ancillary brain blood flow analyses play a larger role in the neurological determination of death? Can J Anaesth 2011; 57: 927-35

2 Wijdicks EF. The case against confirmatory tests for determining brain death in adults. Neurology 2011; 75: 77-83

3 Escudero D, Otero J, Muniz G, et al. The bispectral index scale: its use in the detection of brain death. Transplant Proc 2005; 37: 3661-3

4 Monteiro LM, Bollen CW, van Huffelen AC, Ackerstaff RG, Jansen NJ, van Vught AJ. Transcranial Doppler ultrasonography to confirm brain death: a meta-analysis. Intensive Care Med 2006; 32: 1937-44

5 Schmidt EA, Czosnyka Z, Momjian S, Czosnyka M, Bech RA, Pickard JD. Intracranial baroreflex yielding an early cushing response in human. Acta Neurochir Suppl 2005; 95: 253-6

6 Smith M. Brain death: time for an international consensus. $\mathrm{Br} J$ Anaesth 2012; 108 (Suppl. 1): i6-9.

doi:10.1093/bja/aes243

\section{Bladder control increases the incidence of urinary retention after epidural analgesia after paediatric orthopaedic surgery}

Editor-Epidural analgesia is an important tool in the management of acute postoperative pain in the paediatric population albeit not devoid of complications, including postoperative urinary retention (POUR). ${ }^{1}$ The incidence of POUR in children is reported between $17 \%{ }^{2}$ and $70 \%{ }^{3}$ As nosocomial urinary infection, although less common in children than in adults, increases after catheterization, ${ }^{4}$ we do not routinely use urinary catheters.

We retrospectively compared the incidence of POUR in two populations of children, toilet-trained or not, following similar types of pelvic surgery, open reduction and acetabuloplasty for DDH (Group 1) and shelf operations (Group 2). This 